International Journal of Pattern Recognition and Artificial Intelligence

Vol. 19, No. 8 (2005) 1041-1057

(C) World Scientific Publishing Company

\title{
DETECTION AND COMPENSATION ALGORITHM FOR BACKLIGHT IMAGES WITH FUZZY LOGIC AND ADAPTIVE COMPENSATION CURVE
}

\author{
CHIUN-LI CHIN* and CHIN-TENG LIN ${ }^{\dagger}$ \\ Department of Electrical and Control Engineering \\ National Chiao-Tung University, Taiwan \\ *ernest.ece89g@nctu.edu.tw \\ ${ }^{\dagger}$ ctlin@mail.nctu.edu.tw
}

\begin{abstract}
This paper presents a new algorithm for detection and compensation of backlight images. The proposed technique attacks the weakness of the conventional backlight image processing methods such as over-saturation, losing contrast and so on. The proposed algorithm consists of two operation phases: detection and compensation phases. In the detection phase, we use the spatial position characteristic and histogram of backlight image to obtain two image indices, which can determine the backlight degree of an image. Fuzzy logic is then used to integrate these two indices into a final backlight index determining the final backlight degree of an image precisely. Second, in the compensation phase, to solve the over-saturation problem that exists usually in conventional image compensation methods, we propose the adaptive compensation-curve scheme to compensate and enhance the brightness of backlight images. The luminance of a backlight image is adjusted according to the compensation curve, which is adapted dynamically according to the backlight degree indicated by the backlight index estimated in the detection phase. The performance of the proposed technique is tested on 100 backlight images covering various kinds of backlight conditions and degrees. The experimental and comparison results clearly show the superiority of the proposed technique.
\end{abstract}

Keywords: Color space; histogram; Gaussian smoothing filter; fuzzy IF-Then rule; membership functions; luminance; over-saturation.

\section{Introduction}

Although most consumer cameras have many appealing features, such as automatic focus, automatic exposure, and automatic white-balance, which enable users to easily take good pictures under various shot conditions, users still have chances of getting backlight images. When taking a picture, we always place the main object of focus into the center of image. Thus, if the luminance difference between the main (foreground) object and the background is high, backlight images are usually produced. The aim of this research is to propose an efficient and high-accuracy technique to enhance the backlight images. In other words, we assume that the photographic object is usually displayed at the center of image in the proposed technique. 
There are many different approaches for detection and compensating backlight images. Morimara proposed an exposure control scheme based on the distribution of luminance for multiple regions of a screen. ${ }^{6}$ Haruki and Kikiuchi proposed a method of dividing a screen into six regions and weighting the luminance data of each region to put emphasis on the center of screen. ${ }^{2}$ Another algorithm for exposure control based on fuzzy logic was proposed by Shimizu et al. ${ }^{8}$ This method combined the HIST distribution with fuzzy logic for the compensation of backlight images. Herein, HIST is defined as the ratio between the number of pixels whose brightness is higher than a threshold value and the total number of pixels in the whole TV picture. HIST distribution is plotted on a graph with a horizontal axis of the threshold value of brightness and a vertical axis of the magnitude of HIST. Its shape depends on the shooting condition. Murakami and Honda proposed an exposure control system using color information of the images to perform well-balanced compensation for both the main object and the background. ${ }^{7}$ Testsuya Kuno and Hiroaki Sugiura proposed a newly developed automatic exposure system for digital still cameras (DSC). ${ }^{5}$ In this paper, special hardware mechanism and circuits were used to implement the exposure system of digital cameras. The hardware circuits have to control the aperture and shutter of digital cameras to avoid conditions of under or over exposure. Hence, it requires extra hardware devices, circuits and control. A two-stage algorithm based on the fuzzy rules introduced in Ref. 8 was discussed in Ref. 3. On the other hand, when the luminance of image is enhanced by some compensation methods, the colors of an image may be distorted. In Refs. 1, 4, 9 and 10, several color image processing and color feature extraction methods were used to preserve or enhance the color information in the compensated image.

Although the aforementioned techniques can improve the backlight mages to some extent, they usually suffer the problems of over-saturation, losing contrast and so on. Also, there are some articles [e.g. Refs. 3 and 7] proposed using clustering method like fuzzy c-means, neural networks, mass fuzzy rules and pixel-bypixel compensation algorithm to detect and compensate the backlight images. This always requires high computation power which is unsuitable for use in the realtime commercial products. In this paper, we propose a new efficient algorithm for detecting and compensating backlight images. The block diagram of the proposed algorithm is shown in Fig. 1. When taking a picture, the backlight effect results from the situation that the main object blocks a light source. This will result in the lightness insufficiency of the main object in a backlight image. Therefore, we have to enhance the lightness of the main object and keep the color information in the image. In the paper, the input backlight images are initially transformed into the Y. I. Q. color space. In this space, the luminance $Y$ and color information are decoupled, where $Y=0.299 R+0.587 G+0.114 B$, since luminance is proportional to the amount of light perceived by the human eyes. After the color space transformation, the entire transformed image is analyzed to extract determining features of backlight degree. The analysis is based on the spatial position relation and histogram of images. We will employ fuzzy logic to infer a backlight index to indicate 


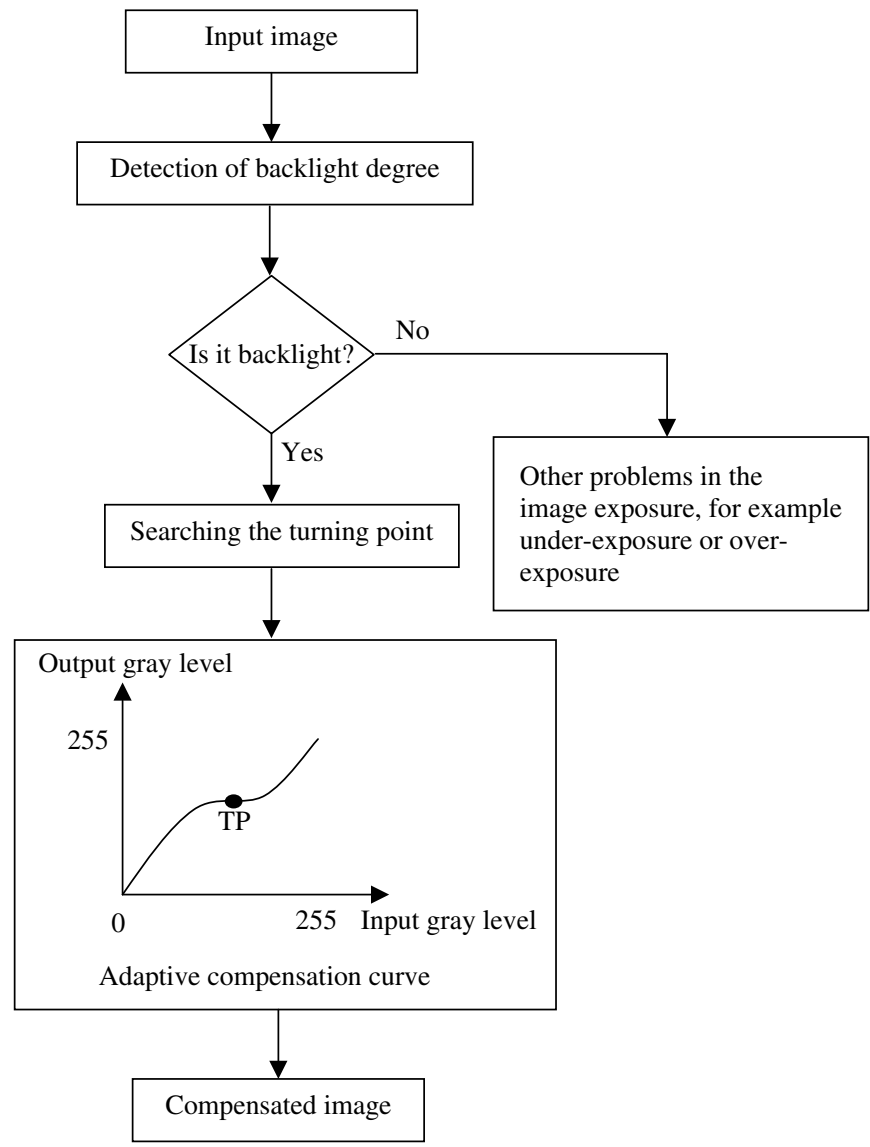

Fig. 1. System architecture for detecting and compensating backlight images.

the backlight degree of an image. If the image is not backlighting, it will be processed by the other auto-exposure methods for solving the possible under-exposure or over-exposure problems and so on. After determining the backlight degree of an image, we will compensate its luminance and keep its contrast according to the estimated backlight degree. To avoid the over-saturation problems, we propose a new adaptive compensation curve scheme for luminance compensation, where the shape of the compensation curve is determined adaptively by its turning point. Finally, the compensated image is transformed back to the R. G. B. color space from the Y. I. Q. color space defined by:

$$
\left[\begin{array}{l}
R \\
G \\
B
\end{array}\right]=\left[\begin{array}{rrr}
1 & 0.956 & 0.620 \\
1 & -0.272 & -0.647 \\
1 & -1.108 & 1.705
\end{array}\right]\left[\begin{array}{l}
Y \\
I \\
Q
\end{array}\right]
$$

which will restore color information of the compensated image. 
This paper is organized as follows. Section 2 describes the proposed backlight detection method, which uses fuzzy logic to determine the backlight degree of an image. Section 3 proposes the backlight compensation method, which utilizes the adaptive compensation curve to compensate the luminance of the backlight image adaptively. Section 4 provides and discusses the experimental results. Finally, Sec. 5 gives conclusions and suggestions for the future works.

\section{Backlight Detection Phase}

When taking a picture, the backlight image results from the illuminant source being at the back of the photographic object and the photographer in front of the object. Therefore, the brightness of photographic object is displayed with dark brightness, and the luminance difference between the main object and the background is high. In this section, we shall derive two indices to detect the backlight degree of an image according to its spatial position characteristic and histogram characteristic, respectively. Fuzzy logic is then used to integrate these two indices into a final backlight index determining the final backlight degree of an image precisely.

To derive the indices for indicating backlight degree of an image, let us observe two representative backlight images and their histograms shown in Fig. 2. In Fig. 2(a), the backlight part gathers at the central part of the image and there

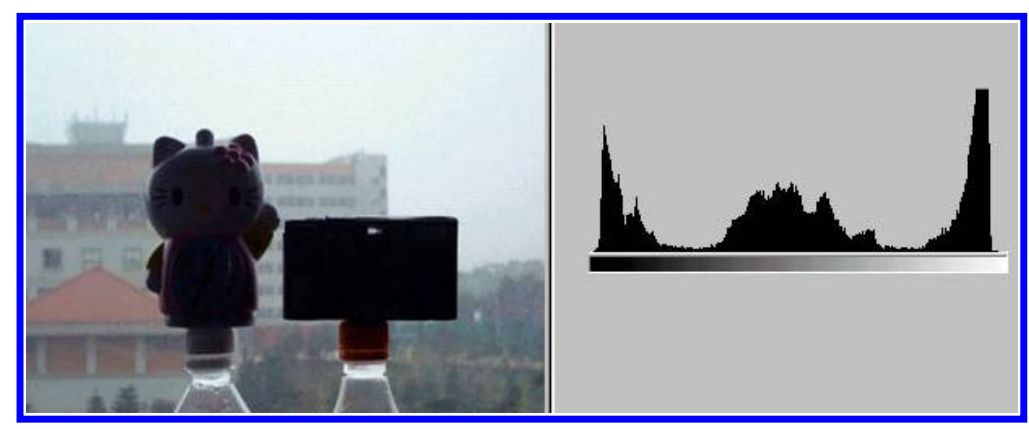

(a)

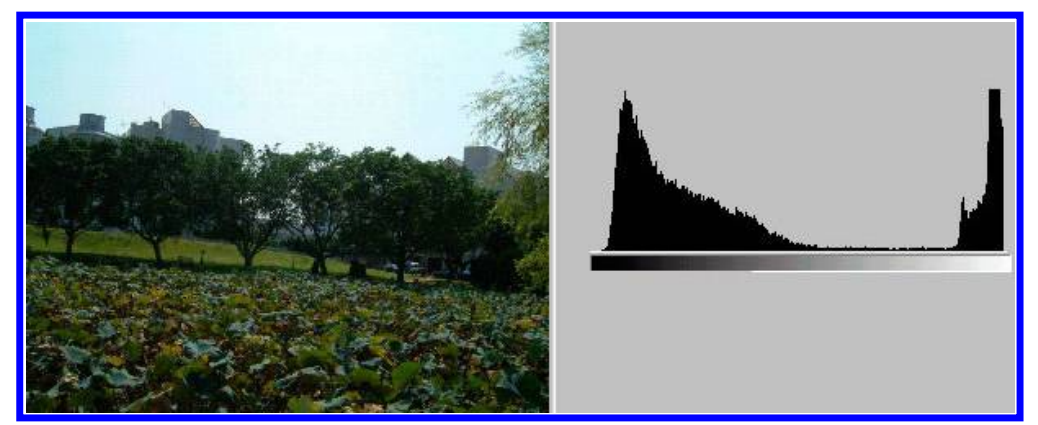

(b)

Fig. 2. Two representative backlight images and their histograms. 
are three groups appearing in the histogram of image. Figure 2(b) shows the general form of backlight images. Its backlight part is also gathered in the central part of the image, but there are only two groups appearing in the histogram of image.

\subsection{Backlight index based on the spatial characteristics of an image}

The main object is usually gathered in the central part of an image when taking a picture. Furthermore, if the image has backlight problems, the luminance of the main object is low and that of the background is high. Therefore, using the spatial characteristics of an image, we can find the index to represent the backlight degree of the image. To do this, we first use a spatial position segmentation method to divide an image into several areas. Then, according to the strength of area-average luminance, they can be merged into two areas; one is background area and the other is main object area. The difference of average luminance between the background area and the main object forms an effective index to indicate the backlight degree of the analyzed image.

In the proposed scheme, we divide an image into five areas as shown in Fig. 3. Since the photographic object is usually displayed in the center of an image when taking a picture, we leave out a fourth part except for the main body in the top/bottom and left/right, respectively, and leave a bigger region, one half of the whole image for the main body. The R3 and R5 areas in Fig. 3 are really photographic objects, and the R1 area is truly a background section in the backlight image. Then, we can use maximum and minimum functions to determine whether the $\mathrm{R} 2$ and $\mathrm{R} 4$ areas are the background parts or the main-object parts by defining the index $B_{\text {image }}$ below:

$$
\begin{aligned}
B_{\text {image }}= & T\left(\left[M R_{1}+\max \left(M R_{2}, M R_{4}\right)\right] / 2\right. \\
& \left.-\left[M R_{3}+M R_{5}+\min \left(M R_{2}, M R_{4}\right)\right] / 3\right),
\end{aligned}
$$

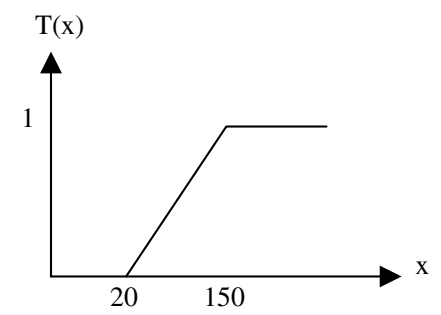

where $M R_{i}$ is the average value of gray level of each area and $T(\cdot)$ is a membership function which converts $B_{\text {image }}$ into a fuzzy degree. The higher this index value, the higher is the backlight degree of image as well. This index value is obtained 


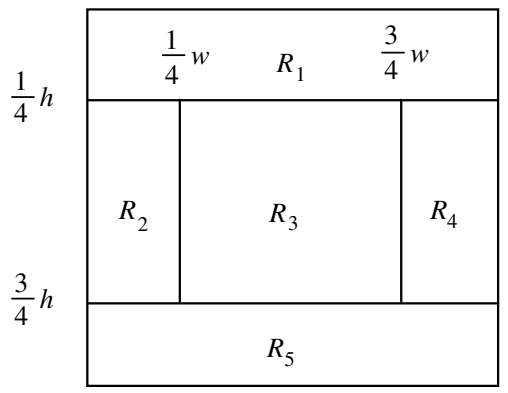

Fig. 3. The division of a backlight image.

from the relation of the spatial position of object area and background area in the backlight image.

\subsection{Backlight index based on the histogram characteristics of an image}

In the above subsection, the $B_{\text {image }}$ index is obtained from the average luminance of different spatial parts of a backlight image. Therefore, it is not considered for the probability distribution of image lightness. In this subsection, we shall derive the second index indicating the backlight degree of an image based on its histogram. Figure 4 shows the representative histogram of a backlight image. This figure illustrates that the backlight image will show the two groups (peak areas) distinctly in the histogram; one group is distributed over high brightness area and the other is distributed over low brightness area. Hence, we can separate the histogram into three areas according to two threshold values T1 and T2. In our scheme, the value of $\mathrm{T} 1$ is set as 60 according to the property of human vision perception stating that the brightness under 60 gray-level will be regarded as darkness. ${ }^{10}$ According to the reason of definition $\mathrm{T} 1$, we use the value " 60 " to be the basis for the definition of T2. Thus, Subtracting 61 from the maximum intensity value of the image gives the value of T2. Herein, the reason that we add one to that value is just to increase the tolerance of T2. And, we reasonably assume that the maximum brightness value is

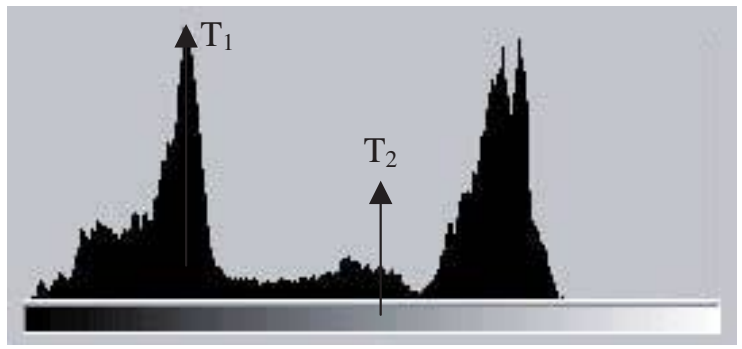

Fig. 4. The representative histogram of backlight images. 
greater than 61 according to our experiments; otherwise the histogram of an image with the maximum brightness value less than or equal to 61 will not appear to be a two-cluster distribution.

According to these two threshold values T1 and T2, we can define the second index, $B_{\text {hist }}$, determining the backlight degree of an image:

$$
B_{\text {hist }}=T\left(\sum_{j=T_{1}}^{T_{2}} p\left(r_{j}\right)\right),
$$

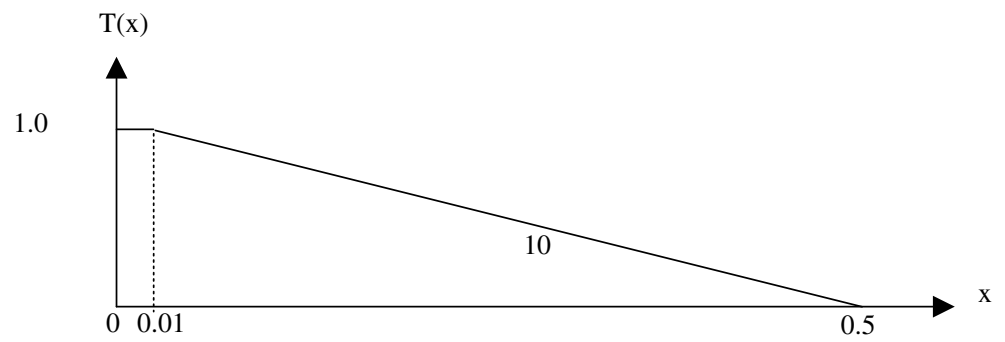

where $p\left(r_{j}\right)=\frac{n_{j}}{n}$ is the probability of the $j$ th gray level, where $n$ is the total number of pixels in the image and $n_{j}$ is the number of times this level appears in the image, and $T(\cdot)$ is a membership function which converts $B_{\text {hist }}$ into a fuzzy degree. This index determines the close degree of the two groups in the histogram. When the two clusters are close, the sum of probability of all gray-levels between them will be very high. It shows that $B_{\text {hist }}$ has a lower value. Thus, the analyzed image has a lower backlight degree. On the contrary, higher $B_{\text {hist }}$ value represents higher backlight degree of the image.

\subsection{Fuzzy integration of two backlight indexes}

In the previous subsections, two indices are derived to indicate the backlight degree of an image based on the average luminance difference of subimages and the histogram, respectively. Due to the various property and content of backlight images, these two indices have different degrees of reliability. Since the $T$ functions used in the definitions of these two indices convert the indices into fuzzy degrees, we shall apply the fuzzy inference technique to fuse these indices ( $B_{\text {image }}$ and $B_{\text {hist }}$ ) and produce a more reliable backlight index, $B_{\mathrm{F}}$, representing the backlight degrees of various kinds of backlight images.

The fuzzy inference rules are characterized by a collection of fuzzy IF-THEN rules in which the preconditions and consequents involve linguistic variables. In the proposed fuzzy inference scheme for backlight detection, there are two input variables, $B_{\text {image }}$ and $B_{\text {hist }}$, and one output variable $B_{\mathrm{F}}$. Each variable has three fuzzy terms (sets), S (small), M (medium), and B (Big). An identical membership function is used for the fuzzy terms of $B_{\text {image }}, B_{\text {hist }}$ and $B_{\mathrm{F}}$ as shown in Fig. 5, when the index $B_{\text {h.i.f. }}$ of figure is an abbreviation, which can be $B_{\text {hist }}, B_{\text {image }}$ 


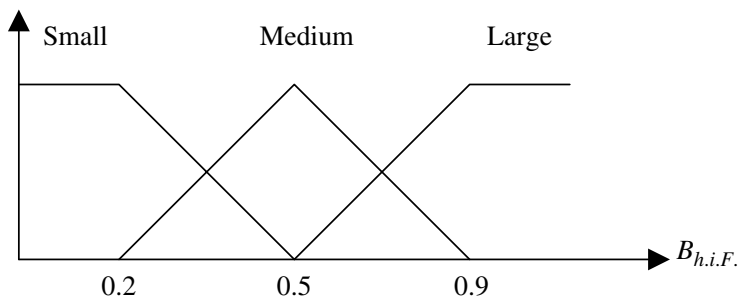

Fig. 5. Membership functions of the fuzzy terms of $B_{\text {hist }}, B_{\text {image }}$ and $B_{\mathrm{F}}$.

or $B_{\mathrm{F}}$. Therefore, nine fuzzy inference rules are used here. Two representative rules are enumerated as follows:

1. If $B_{\text {image }}$ is $\mathrm{S}$ and $B_{\text {hist }}$ is $\mathrm{S}$ then $B_{\mathrm{F}}$ is $\mathrm{S}$.

2. If $B_{\text {image }}$ is $\mathrm{M}$ and $B_{\text {hist }}$ is $\mathrm{M}$ then $B_{\mathrm{F}}$ is L.

Based on the center of area (COA) defuzzification method, we can obtain the $B_{\mathrm{F}}$ value corresponding to two given $B_{\text {image }}$ and $B_{\text {hist }}$ values through fuzzy inference. The inferred $B_{\mathrm{F}}$ value represents the final estimated backlight degree of the analyzed image in the detection phase of the proposed scheme. In the next section, this index will be used to find a proper turning point on the adaptive compensation curve in the backlight image compensation phase of our scheme.

\section{Backlight Compensation Phase}

In the previous section, we proposed a procedure to detect the backlight degree of an image. After the backlight degree is detected, we can then compensate the backlight image according to the detected backlight degree. In general, ${ }^{7}$ this is achieved by adding a compensation value to each brightness value of the target image. Although the brightness of the backlight part in the image can be emphasized by this compensation value, the brightness of the background part might be out of the maximum intensity range of image, causing the over-saturation phenomena. To solve this problem, we propose a new image compensation scheme based on adaptive compensation curves. This curve can be automatically adapted to adjust the compensation amount according to the backlight degree of the image. After compensation, the compensated image will keep the characteristics of the original image and the brightness of the backlight part of image will be increased properly.

The proposal compensation curve is shown in Fig. 6. It has a turning point, which separates the compensation curve into the upper and lower parts. The lower curve will gradually increase the dark-part brightness of the input image and the upper curve will keep light-part brightness of the input image. By controlling this curve curvature properly, we can compensate the lightness of backlight image successfully without causing the over-saturation problem. To ease the computational complexity, we use parabolic curves to construct the compensation curve. The upper 
Output gray level

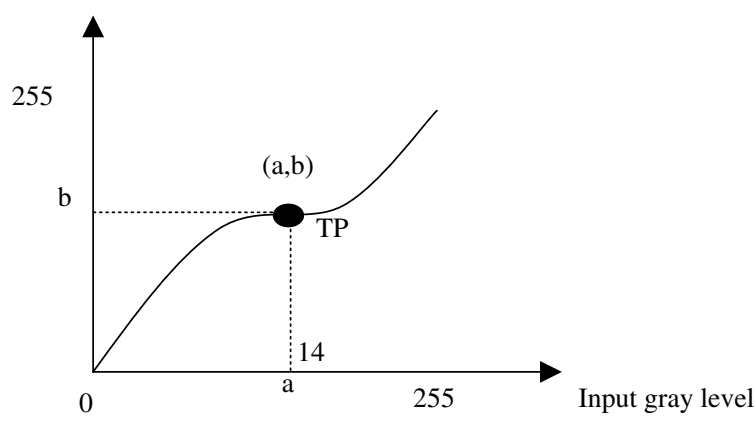

Fig. 6. Adaptive curve for image compensation.

and lower curves are constructed by upward and downward parabolic curves, respectively, which are described by Eqs. (1) and (2):

$$
\begin{aligned}
& f(x)=\frac{-b}{a^{2}}(x-a)^{2}+b \\
& f(x)=\frac{(255-b)}{(255-a)^{2}}(x-a)^{2}+b,
\end{aligned}
$$

where the coordinate of the turning point (i.e. the apex of these two curves) is $(a, b)$, which can be obtained from the estimated backlight degree $\left(B_{\mathrm{F}}\right)$ of the image in the backlight detection phase as described in the following procedure.

To determine the turning point in Fig. 6, let us observe the histograms of two representative backlight images shown in Figs. 7(a) and 7(b). There are apparently two groups in Fig. 7(a). The first group (group A) has lower brightness, and the second group (group B) has higher brightness. We can utilize this characteristic to get the turning point. At first, we calculate the average values of groups A and B, called $L_{m}$ and $H_{m}$, respectively. We use the following steps to obtain the values of $L_{m}$ and $H_{m}$.

Step 1: Using Gaussian smoothing filter to smooth the histogram of the whole image.

Step 2: Calculating a series $\left\{a_{j}\right\}_{j=0}^{L-1}=\left\{a_{0}, a_{1}, \ldots, a_{j}, \ldots, a_{L-1}\right\}$ and series $b$ by the following equations:

$$
\begin{gathered}
b=\left\{a b s\left(a_{i+1}-a_{i}\right)\right\}_{0}^{L-1}=\left\{\left|a_{1}-a_{0}\right|,\left|a_{2}-a_{1}\right|, \ldots,\left|a_{L-1}-a_{L-2}\right|\right\} \\
a_{j}=\left\{\begin{array}{ll}
j & \text { if } 0<P\left(r_{j}\right) \leq T H \\
0 & P\left(r_{j}\right)>T H
\end{array}, j=0,1,2, \ldots, L-1,\right.
\end{gathered}
$$

where $\mathrm{TH}$ is a threshold which is around 0.0013 according to our experimental study. 


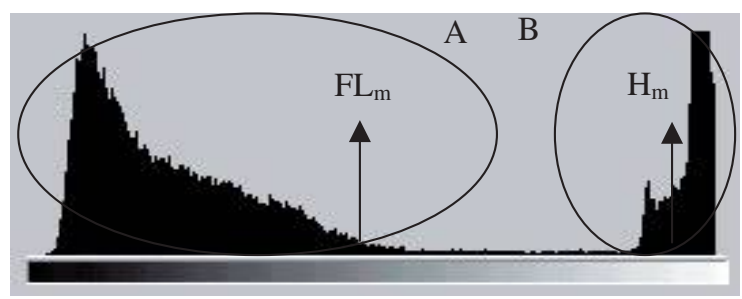

(a)

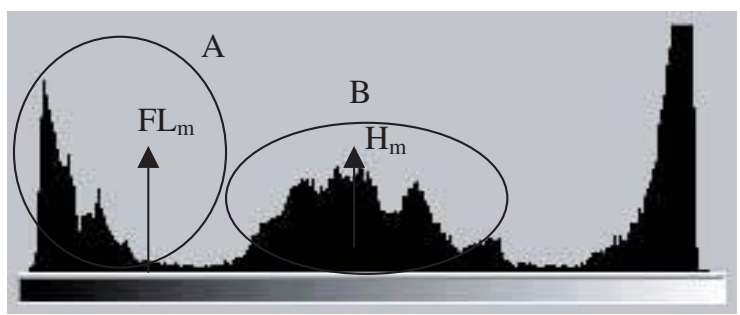

(b)

Fig. 7. Histograms of two representative backlight images.

Step 3: According to series $a$ and $b$ to obtain the $T$ set, $T=\left\{T_{1}, T_{2}, T_{3}, \ldots\right\}$, which contains the start and end points of groups A and B:

$$
T_{k}= \begin{cases}j & \text { if } b_{i}=a_{j} \text { and } b_{i}=a_{j} \neq 0 \\ i+1 & \text { if } b_{i}=a_{i+1}\end{cases}
$$

where $k=1,2,3, \ldots, i=0,1,2, \ldots, L-2$, and $j=0,1,2, \ldots, L-1$, where $L$ is the maximum intensity value of image.

Step 4: Calculating the values of $L_{m}$ and $H_{m}$ by $T$ set.

$$
L_{m}=\frac{\sum_{j=T_{1}}^{T_{2}} P\left(r_{j}\right)^{*} j}{\sum_{j=T_{1}}^{T_{2}} p\left(r_{j}\right)}, \text { and } H_{m}=\frac{\sum_{j=T_{3}}^{T_{4}} P\left(r_{j}\right)^{*} j}{\sum_{j=T_{3}}^{T_{4}} p\left(r_{j}\right)} .
$$

Next, we will utilize the $B_{\mathrm{F}}$ index obtained in the backlight detection phase to determine the final $L_{m}$ value, called $F L_{m}$, by

$$
F L_{m}=L_{m}+L_{m}^{*} B_{\mathrm{F}} .
$$

This value represents the highest gray value of dark area in the image histogram. We shall stretch this value to $H_{m}$ such that the brightness of the dark area in the image will be enhanced. To do this, we let the $y$ coordinate of the turning point of the compensation curve be the value $H_{m}$ and the $x$-coordinate be the value $F L_{m}$, i.e. $(a, b)=\left(F L_{m}, H_{m}\right)$, in Eqs. (1), (2) and Fig. 6. This also decides the shape of the whole compensation curve.

Figure $7(\mathrm{~b})$ shows the histogram of another representative backlight image. There are three groups in this histogram, representing low brightness, median brightness and high brightness areas in the image, respectively. The median and 
high brightness areas correspond to the background of the image, and the low brightness area corresponds to the backlight part of the image. Also from Fig. 7(b), we discover that the histogram distribution among median brightness area and high brightness area is uniform. Thus, we can stretch the backlight part of the image from the low brightness area to the median brightness area. Hence, by identifying the low brightness area as group A, and the median brightness area as group B, the procedure proposed before based on the histogram of Fig. 7(a) can be applied here to determine the turning point $(a, b)$ of the compensation curve.

\section{Experimental Results}

The performance of the proposed backlight detection and compensation scheme is tested in this section. All the experiments were performed on a personal computer with Pentium III 700. The testing image base consists of 100 backlight images. Figures $8(\mathrm{a})$ to $8(\mathrm{~d})$ show some typical images in our testing image database. These images were captured by the FinePix 6800Zoom of Fujifilm camera and the resolution of each image is $640 * 480$ pixels. In the following, we shall demonstrate the performance of the proposed scheme based on the four images in Figs. 8(a) to 8(d). Performance comparisons are also made by applying the method in Ref. 7 on the same images. In Ref. 7, the author uses fuzzy logic method to infer a compensation value C. For inferring this value, they define four fuzzy indices extracted from backlight image. These indices are average luminance of a screen (V), Importance of the background (BI), Backlight degree (B), and Excessive frontlighting degree (EF). The four fuzzy indices will produce 21 rules and their final compensation algorithm is represented as follows:

(a) Luminance difference $=$ Average luminance of a image - reference value

(b) Luminance of the pixel after compensation = Luminance of the pixel before compensation - luminance difference.

Figures $8(\mathrm{a})$ to $8(\mathrm{~d})$ show four original backlight images, where the first two images were captured indoor and the rest two were captured outdoor. We applied our method and the method in Ref. 7, respectively, on these four images to obtain the corresponding compensated images in Figs. 8(e) to 8(l). Figures 8(e) to 8(h) show the compensated images by our method. Figures. 8(i) to 8(l) show the compensated images by the method proposed by M. Murakami and N. Honda in Ref. 7 where a proper fixed gray value was added to each brightness value of the whole image for brightness compensation. We noticed that the over-saturation problems occurred in Figs. 8(i) to 8(1). In these compensated images, although the brightness of the backlight part in the image has been enhanced, the brightness of the background part became too high and some detailed information was lost. For example, let us observe Figs. 8(b), 8(f) and 8(j). In Fig. 8(b), the original backlight image has the lowest brightness in the central object part, but has normal brightness in the background part. In the compensated image in Fig. 8(f), the brightness of the 


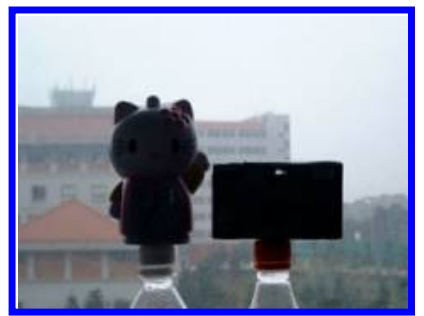

(a)

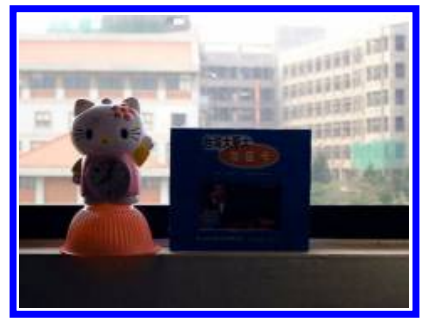

(b)

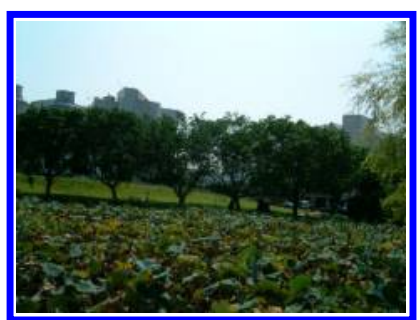

(c)

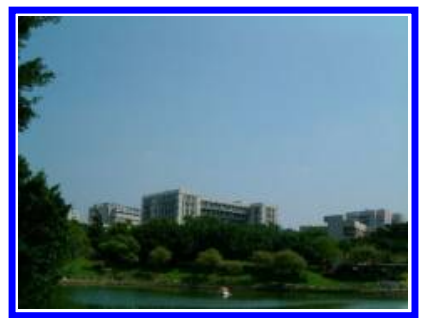

(d)

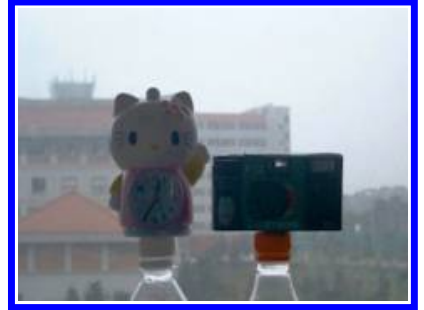

(e)

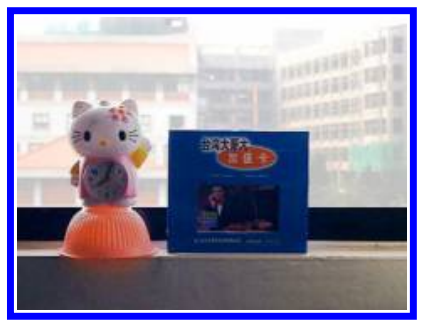

(f)

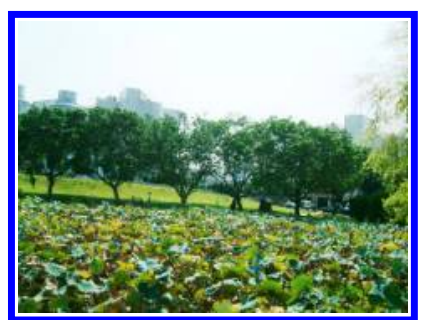

(g)

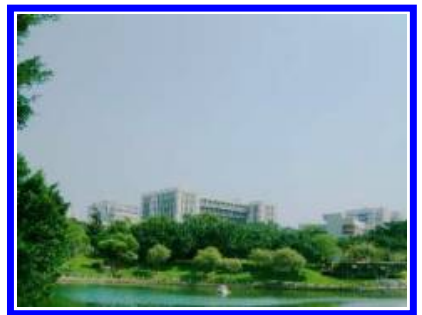

(h)

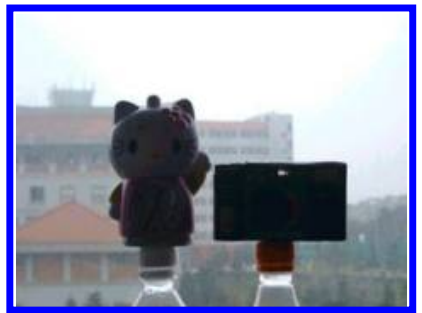

(i)

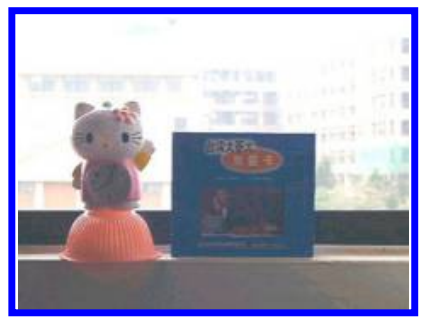

(j)

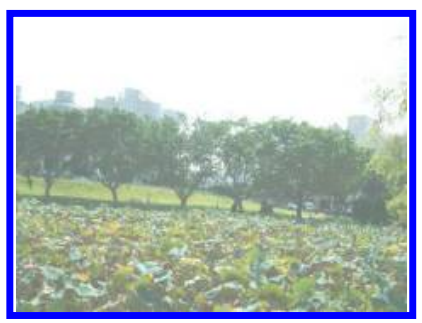

(k)

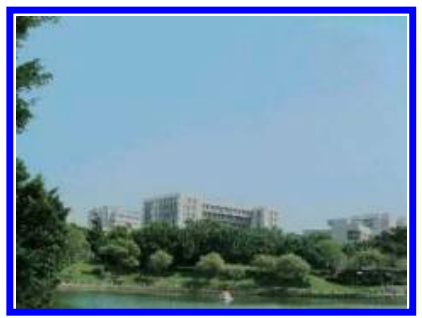

(l)

Fig. 8. (a)-(d) Original images, (e)-(h) compensated images by our method, and (i)-(l) compensated images by the method in Ref. 7 .

backlight part is enhanced properly. But in the compensated image in Fig. 8(j), the brightness of the backlight part is obviously saturated. Therefore, the luminance in the background of this image seems unnatural to human vision perception. The results clearly demonstrate the superiority of the proposed backlight compensation technique. Subsequently, we compare our method to the histogram equalization 


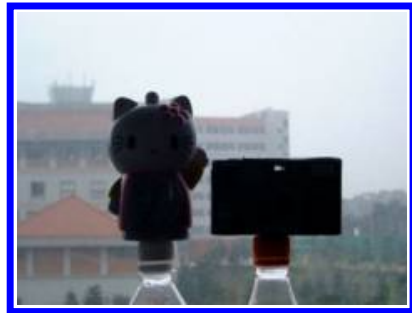

(a)

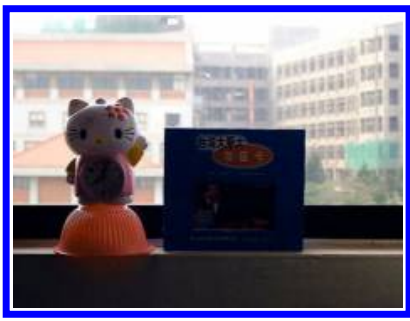

(b)

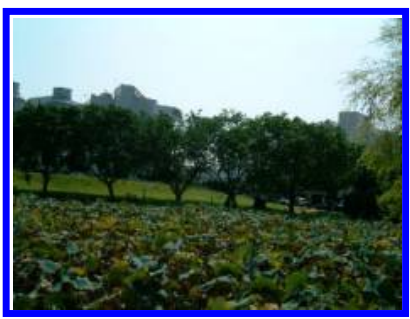

(c)

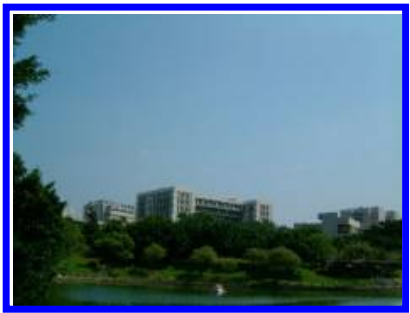

(d)

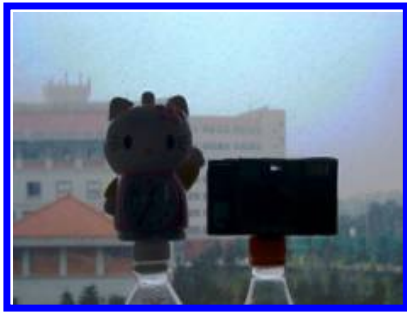

(e)

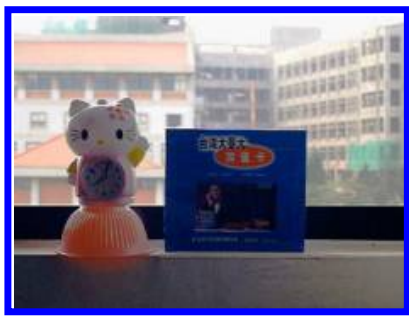

(f)

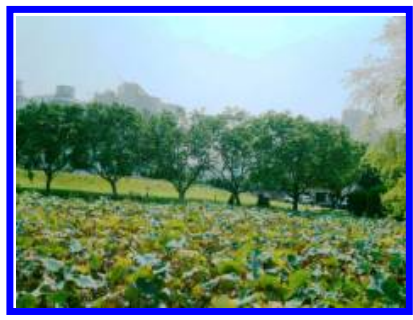

(g)

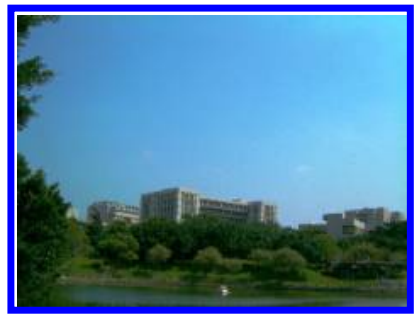

(h)

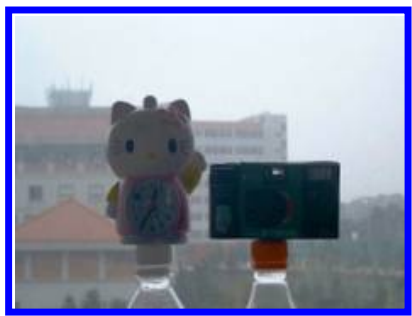

(i)

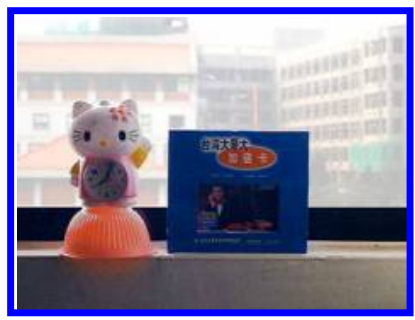

(j)

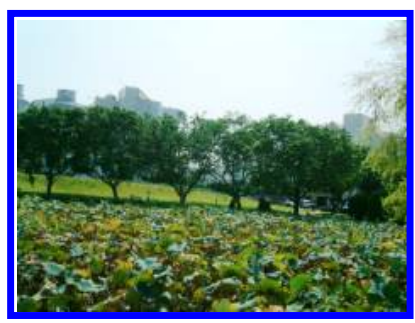

(k)

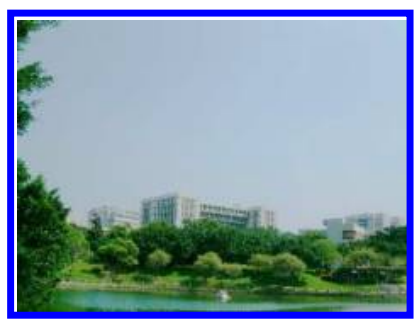

(l)

Fig. 9. (a)-(d) Four original backlight images, (e)-(h) compensated images by the histogram equalization method, and (i)-(l) compensated images by our proposed method.

method. Figures 9(a) to 9(d) are four original backlight images and Figs. 9(e) to 9(h) show the compensated images by the histogram equalization method. These processed images appear to be over-compensated. Namely, it will produce an effect of intensity saturation in some areas. This defeat is appeared because of the process attempted to merge the adjacent gray levels together in order to flatten the 
occurred. This is because we compensate only for the main object with scanty light, we do not destroy the original information of the object with the normal light. Thus for images after processing, the brightness of the main object will be enhanced, and the remained section will also not be destroyed.

\section{Conclusions}

In this paper, we proposed a new algorithm for detection and compensation of backlight images. We derived a new backlight index through a fuzzy inference mechanism to represent the backlight degree of an image. The brightness of the backlight image was then compensated according to a compensation curve determined by the backlight index adaptively. The proposed algorithm was tested on 100 backlight images, which contained diverse environments with various backlight degrees. Experimental results and performance comparisons clearly indicated the superiority of the proposed scheme, especially in solving the over-saturation problems that existed in the current backlight compensation approaches. We now focus on integrating the proposed backlight detection and compensation scheme with the solutions of other automatic exposure problems into one intelligent algorithm for applications in real-world products such as digital still cameras and digital camcorder.

\section{References}

1. R. C. Gonazlea and R. E. Woods, Digital Image Processing, 2nd ed. (Addison-Wesley, MA, Reading, 1992).

2. T. Haruki and K. Kikiuchi, Video camera system using fuzzy logic, IEEE Trans. Consumer Electron. 38(3) (1992) 624-634.

3. J. Q. Huang, New technique for digital backlight image compensation, Master thesis, Department of Computer Science and Information Engineering, Chung Hua University, Taiwan (June 2001).

4. A. K. Jain, Fundamentals of Digital Image Processing (Englewood Cliffs, PrenticeHall, NJ, 1989).

-5. T. Kuno and H. Sugiura, A new automatic exposure system for digital still cameras, IEEE Trans. Consumer Electron. 44(1) (1998) 192-199.

6. A. Morimura, K. Uomori, Y. Kitamura, A. Fujioka, J. Harada, S. Iwamura and M. Hirota, A digital video camera system, IEEE Trans. Consumer Electron. 36(4) (1990) 886-875.

7. M. Murakami and N. Honda, An exposure control system of video cameras based on fuzzy logic using color information, in Proc. Fifth IEEE Int. Conf. Fuzzy Systems 3 (1996) 2181-2187.

8. S. Shimizu, T. Kondo, T. Koashi, M. Tsurata and T. Komura, A new algorithm for exposure control based on fuzzy logic for video cameras, IEEE Trans. Consumer Electron. 38(3) (1992) 617-623.

9. C. M. Tsai and H. J. Lee, Binarization of color document images via luminance and saturation color features, IEEE Trans. Imag. Process. 11(4) (2002). 
10. C. C. Yang and J. J. Rodriguea, Efficient luminance and saturation processing techniques for color images, J. Vis. Commun. Imag. Represent 3(3) (1997) 263-277.

11. J. Zimmerman, S. Pizer, E. Staab, E. Perry, W. McCartney and B. Brenton, Evaluation of the effectiveness of adaptive histogram equalization for contrast enhancement, IEEE Trans. Med. Imag. (1988) 304-312.

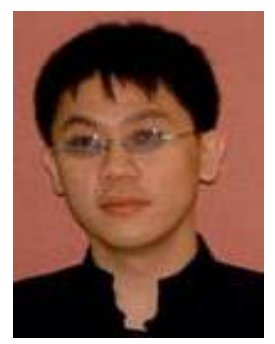

Chiun-Li Chin received the B.S. and M.S. degrees from the Department of Computer Science and Information Engineering, Chung-Hua University, HsinChu, in 1998 and 2000, respectively. He is currently pursuing the Ph.D. in Department of Electrical and Control Engineering, National Chiao Tung University.

His research interests include fuzzy inference, neural network, color image processing, computer vision, pattern recognition and document image analysis. 


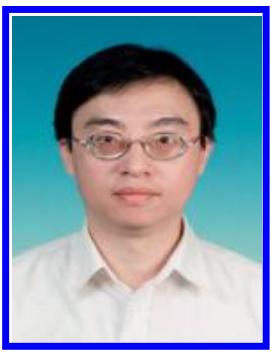

Chin-Teng Lin (S'88-

M'91-SM'99-F'04) received the B.S. degree in control engineering from the National Chiao-Tung University (NCTU), Hsinchu, Taiwan, R.O.C., in 1986, and the M.S.E.E. and $\mathrm{Ph} . \mathrm{D}$. degrees in electrical engineering from Purdue University, West Lafayette, IN, in 1989 and 1992, respectively. Since August 1992, he has been with the College of Electrical Engineering and Computer Science, National Chiao-Tung University, where he is currently the Associate Dean of the college and a Professor of Electrical and Control Engineering Department. He has also served as the Director of Brain Research Center, NCTU Branch, University System of Taiwan since September 2003. He served as the Director of the Research and Development Office of the National Chiao-Tung University from 1998 to 2000, and the Chairman of the Electrical and Control Engineering Department from 2000 to 2003.

$\mathrm{He}$ is the co-author of Neural Fuzzy Systems - A Neuro-Fuzzy Synergism to Intelligent Systems (Prentice Hall), and the author of Neural Fuzzy Control Systems with Structure and Parameter Learning (World Scientific, 1994). He has also published over 80 journal papers in the areas of neural networks, fuzzy systems, multimedia hardware/software, and soft computing, including 60 IEEE journal papers. Dr. Lin is a member of Tau Beta Pi, Eta Kappa $\mathrm{Nu}$, and Phi Kappa Phi honorary societies. He is also a member of the IEEE Circuit and Systems Society (CASS), the IEEE Neural Network Society, the IEEE Computer Society, the IEEE Robotics and Automation Society, and the IEEE System, Man, and Cybernetics Society.

He currently serves as an Associate Editor of the IEEE Transactions on Circuits and Systems: I, IEEE Transactions on Circuits and Systems: II, Int. J. Speech Technology, and the Journal of Automatica.

His current research interests are in neural networks, fuzzy systems, cellular neural networks (CNN), fuzzy neural networks (FNN), neural engineering, algorithms and VLSI design for pattern recognition, intelligent control, and multimedia (including image/video and speech/audio) signal processing, and intelligent transportation system (ITS). 
This article has been cited by:

1. Cheng-Jian Lin, Yong-Cheng Liu. 2009. Image backlight compensation using neuro-fuzzy networks with immune particle swarm optimization. Expert Systems with Applications 36:3, 5212-5220. [CrossRef] 